\title{
Innover, collaborer, apprendre L'initiative École en réseau dans la mise en pratique de l'apprentissage à distance des élèves et des enseignants
}

\section{Innovate, collaborate, learn: the École en réseau initiative in the practice of distance learning for students and teachers}

\section{Innovar, colaborar, aprender: la iniciativa École en réseau en la práctica del aprendizaje a distancia para alumnos y profesores}

https://doi.org/10.52358/mm.vi8.187

Sophie Nadeau-Tremblay, enseignante ressource

École en réseau, Canada

sophie.nadeaut@eer.qc.ca

Jessica Métivier, conseillère pédagogique

Cégep de Sainte-Foy, Canada

imetivier@csfoy.ca

RÉSUMÉ

L'initiative École en réseau (ÉER) soutient des enseignants pour qu'ils collaborent à des projets interclasses pour enrichir et diversifier l'environnement d'apprentissage par le numérique (Allaire et al., 2008). Les pratiques mises en œuvre ont permis de développer dans l'ÉER une expertise dans le travail en réseau et l'apprentissage connecté (Bruillard et al., 2021). Au printemps 2020, la situation mondiale a forcé le monde éducatif à revoir sa manière de soutenir les élèves. La transposition de la classe en réseau à la classe à distance a été aisée pour les enseignants de l'ÉER. L'article relate l'innovation, la collaboration et l'apprentissage en réseau de cette forme pédagogique particulière mise en œuvre par l'ÉER pour la formation à distance des enseignants et l'enseignement à distance des ordres d'enseignement préscolaire et primaire. 
Mots-clés : apprentissage en réseau, enseignants du préscolaire et du primaire, pandémie de COVID-19, développement professionnel

\section{ABSTRACT}

The École en Réseau (ÉER) initiative supports teachers to collaborate on inter-class projects to enrich and diversify the digital learning environment (Allaire et al., 2008). The practices implemented have made it possible to develop expertise in networking and connected learning in l'ÉER (Bruillard et al., 2021). In the spring of 2020, the global situation forced the educational world to rethink their ways of supporting students. Transposing from the networked classroom to the remote classroom was easy for l'ÉER teachers. The article chronicles the innovation, collaboration and network learning of this particular form of teaching implemented by ÉER for distance training of teachers and distance education in preschool and primary schools.

Keywords: network learning, preschool/primary teachers, COVID-19 pandemic, professional development

\section{RESUMEN}

La iniciativa École en Réseau (ÉER) apoya a los profesores para que colaboren en proyectos entre clases para enriquecer y diversificar el entorno de aprendizaje digital (Allaire et al., 2008). Las prácticas implementadas han hecho posible desarrollar experiencia en redes y aprendizaje conectado en l'ÉER (Bruillard et al., 2021). En la primavera de 2020, la situación global obligó al mundo educativo a repensar sus formas de apoyar a los estudiantes. La transición del aula en red al aula remota fue fácil para los profesores de l'ÉER. El artículo narra la innovación, la colaboración y el aprendizaje en red de esta forma particular de enseñanza implementada por ÉER para la formación a distancia de docentes y la educación a distancia en escuelas preescolares y primarias.

Palabras clave: aprendizaje en red, docentes de preescolar/primaria, pandemia COVID-19, desarrollo profesional

\section{École en réseau : quelques repères historiques}

Développer une culture de collaboration entre élèves et enseignants de classes distantes autour d'objets d'apprentissage du Programme de formation de l'école québécoise (PFÉQ). Tel était l'objectif du CEFRIO, une équipe de chercheurs universitaires préoccupée de la vitalité des petites écoles qui, avec la collaboration du ministère de l'Éducation du Québec, ont créé l'École éloignée en réseau (ÉÉR) au début des années 2000 (Laferrière et al., 2006; Laferrière et al., 2004). En partenariat avec les milieux de pratique, ils ont structuré une manière différente d'enseigner et d'apprendre en réseau en s'appuyant sur des outils technologiques soutenant la collaboration à l'oral (visioconférence) et à l'écrit (outil d'écriture collaborative). Ainsi, les classes des écoles participantes s'ouvrent à d'autres afin d'enrichir, à distance, l'environnement éducatif. Au fil des années, le réseau de l'ÉÉR s'est élargi avec des classes de grandes écoles et de milieux urbains désireuses d'enrichir eux aussi l'environnement d'apprentissage de leurs élèves; l'École éloignée en réseau est ainsi devenue l'École en réseau (ÉER) et des pays 
francophones s'en sont inspiré pour mettre en place leur propre modèle (Bruillard et al., 2021). Au Québec, l'initiative ÉER bénéficie de l'appui du ministère de l'Éducation depuis sa création et constitue une mesure du Plan d'action numérique en éducation (Ministère de l'Éducation, du Loisir et du Sport, 2018).

Les enseignants participants notent deux indices de la portée de l'apprentissage en réseau : 1) des interactions signifiantes entre élèves dans une démarche d'investigation collective basée sur la coélaboration de connaissances (Allaire et Laferrière, 2013; Scardamalia et Bereiter, 2010); 2) la collaboration entre enseignants au moyen de projets novateurs et la présence en réseau de partenaires extrascolaires, tels des musées et organismes scientifiques, qui participent à ces activités interclasses.

La pandémie de COVID-19 a forcé le monde scolaire à revoir ses pratiques (Bozkurt et al., 2020) et le développement de la compétence numérique (Ministère de l'Éducation et de l'Enseignement supérieur, 2019) a été grandement sollicité dans les derniers mois. Pour les enseignants qui travaillaient déjà en réseau avec d'autres classes, la posture pédagogique exigée par le basculement vers une classe à distance avec des élèves du préscolaire et du primaire a été facilitée (Pelletier et al., 2021). Dans les classes, l'appropriation technologique était déjà effectuée, les élèves étant habilités à travailler en visioconférence et par une collaboration asynchrone à l'écrit sous forme numérique. Sur le plan pédagogique, la mobilisation des élèves dans des projets signifiants et engageants est facilitée par le travail en réseau. L'expertise ancrée dans la pratique des milieux éducatifs s'est inscrite en continuité avec les pratiques antérieures devant la nécessité de travailler à distance avec des élèves en début de scolarisation. Les activités vécues depuis plus de 15 ans par des enseignants du préscolaire et du primaire de ÉER ont permis de transposer le travail interclasses à un contexte de classe à distance. Du printemps à l'automne 2020, il s'avère pertinent de porter un regard sur les actions de l'initiative ÉER. Les angles de l'innovation, de la collaboration et de l'apprentissage en réseau sont retenus comme facilitateurs de l'apprentissage à distance des élèves du préscolaire et du primaire, et du développement professionnel des enseignants.

\section{Innover}

À la mi-mars 2020, le premier ministre du Québec met sur pause le système éducatif québécois pour deux semaines. Le 22 mars 2020, il annonce une fermeture de tous les établissements scolaires jusqu'au $1^{\text {er }}$ mai et mentionne que, dans les semaines à venir, le ministère de l'Éducation va acheminer des listes de travaux pour aider les jeunes à continuer leurs apprentissages. Dès lors, des enseignants de l'ÉER entrent spontanément en contact avec leurs élèves par visioconférence afin d'assurer une continuité des apprentissages. Bien que le ministre de l'Éducation n'exige pas cette modalité à ce moment, les enseignants constatent que le contact plus direct avec les élèves, et surtout le fait de les rassembler en classe virtuelle, est facilitant : ils perçoivent plus facilement leurs besoins, la communication est plus aisée qu'au téléphone (modalité alors proposée par le gouvernement) et, surtout, les élèves peuvent briser l'isolement auquel ils sont soumis par le confinement exigé.

C'est en s'appuyant sur l'expertise développée dans le travail interclasses que ces enseignants ont tout naturellement innové pour l'adapter au contexte de la classe à distance. Cette pratique, fort émergente à l'éducation préscolaire et à l'enseignement primaire, a rassuré les élèves et les parents qui ont été nombreux à acheminer des remerciements à l'enseignant de leur enfant en maintenant ainsi un contact direct et signifiant, et de les accompagner de façon proximale dans la continuité et la poursuite de leurs apprentissages. 
Après quelques jours à discuter d'éléments plus affectifs, les enseignants ont senti le besoin de soutenir l'apprentissage de leurs élèves. D'ailleurs, plusieurs réclamaient à leur enseignant de vivre des activités « comme à l'école ». Les trousses ministérielles tardant à arriver dans les familles, plusieurs enseignants ont amorcé des activités pédagogiques avec leurs élèves : lire une histoire et en discuter, proposer des activités de résolution de problèmes mathématiques, partager un lien vers une expérience scientifique à faire en famille et discuter des résultats en classe. Dans de nombreux milieux familiaux, les activités proposées ne se vivaient pas qu'avec l'élève concerné : les frères et sœurs d'autres niveaux scolaires y participaient également. Le contact direct répondait au besoin de tous.

Les enseignants ont continué à innover par des propositions didactiques originales. Par exemple, les limites langagières des jeunes du préscolaire et de la $1^{\text {re }}$ année ont amené des enseignants à créer des livres numériques hebdomadairement pour présenter les contenus des trousses ministérielles. Ainsi, plutôt que la tâche d'animation de ces activités ne revienne au parent, l'enfant était en mesure de faire bon nombre d'activités seul et ainsi d'y développer son autonomie. D'autres enseignants ont préparé des enregistrements, chaque semaine, pour la présentation des activités à réaliser à la maison alors que certains animaient ces activités en visioconférence et proposaient des tâches en mode asynchrone aux élèves.

Au début mai, lors du retour en classe des élèves, des enseignants ont imaginé des manières d'assurer le suivi avec tous leurs élèves. De fait, les groupes-classes étaient souvent divisés à cause du nombre restreint d'élèves exigé par local (maximum de 15) et aussi du choix de parents de garder leurs enfants à la maison. Pour les enseignants travaillant déjà en réseau, il s'agissait alors de mettre en place leur classe en réseau composée des élèves en présentiel avec eux, d'autres regroupés dans un local avec un enseignant suppléant ainsi que ceux restés à la maison. Bien qu'exigeant une logistique sur le plan de l'horaire, toutes ces initiatives ont démontré la faisabilité de l'apprentissage à distance au préscolaire et au primaire en maintenant un climat rassembleur autour de la classe. L'expertise développée dans le travail en réseau par des enseignants de l'ÉER leur a permis d'innover dans ce nouveau contexte pédagogique, et ce, en misant le plus possible sur la collaboration entre les élèves, composante au centre du modèle pédagogique de l'ÉER.

\section{Collaborer}

La collaboration est un élément clé du modèle pédagogique de l'ÉER s'articulant autour de trois volets : la création d'une communauté d'apprentissages, la coélaboration de connaissances et la progression du discours (Allaire et Laferrière, 2013; Allaire et Lusignan, 2015; Laferrière et al., 2010). Les élèves sont amenés à collaborer dans leur classe, mais également avec des élèves d'autres classes autour des activités d'investigation collective. Outre la collaboration qui prend forme entre les élèves dans l'ÉER, une collaboration entre enseignants se développe, que ce soit par les interactions dans une activité interclasses, dans celles avec des partenaires extrascolaires ou dans l'élaboration de nouvelles activités coconstruites par des enseignants et des conseillers pédagogiques.

Au printemps 2020, I'Université TÉLUQ, une référence québécoise en formation à distance au postsecondaire, a proposé à l'ÉER d'élaborer conjointement les quatre microprogrammes de la formation J'enseigne à distance pour le préscolaire et le primaire ${ }^{1}$. L'expertise acquise au fil des années dans le travail en réseau ainsi que l'expertise d'accompagnement des enseignants dans l'émergence de l'enseignement à distance ont été mises à profit dans cette collaboration inédite, de même qu'avec le Centre de services scolaire Beauce-Etchemin qui développait les microprogrammes destinés aux

\footnotetext{
${ }^{1}$ https://jenseigneadistance.teluq.ca
} 
enseignants du secondaire. Afin d'ancrer l'utilisation du numérique en classe à distance dans la pédagogie, l'équipe de l'ÉER a proposé de faire appel aux conseillers pédagogiques du Réseau sur le développement des compétences des élèves par l'intégration des technologies (RÉCIT), qui soutiennent l'intégration du numérique dans les classes québécoises, ainsi qu'à un grand nombre d'enseignants qui construisent au fil des jours des modèles d'enseignement à distance. Les douze modules développés dans un partenariat dirigé par l'équipe de l'ÉER prennent donc assises dans les pratiques novatrices expérimentées pendant plusieurs années dans le travail interclasses en réseau ainsi que plus récemment en formation à distance au préscolaire et au primaire. Ils sont une occasion de développement professionnel appuyée sur la pratique.

Toutefois, on ne peut parler de travail interclasses en réseau ou d'enseignement à distance sans évoquer la participation de l'élève dans ces nouvelles façons d'enseigner et d'apprendre. Au fil des ans, l'ÉER a développé une expertise de pointe pour concrétiser et structurer la collaboration interclasses en réseau, tant du point de vue de l'enseignant impliqué que de celui de ses élèves. Ces pratiques collaboratives s'appliquent au sein de communautés d'apprentissage qui soutiennent le traitement de questions complexes, par les élèves et leur enseignant, au moyen de la résolution de problèmes. Cette agentivité mise à profit par les élèves et l'enseignant les amène à s'engager davantage dans leur propre apprentissage. C'est ce qu'on appelle « apprendre en réseau ».

\section{Apprendre en réseau}

« Apprendre en réseau » réfère à la coconstruction des connaissances autour d'un sujet appuyée par des ressources, des partenaires extrascolaires (experts de contenus) et, surtout, d'élèves d'autres classes. Se réalisant en réseau, soit à distance, l'apprentissage en réseau contextualise et facilite le développement des dimensions de la compétence numérique (Ministère de l'Éducation et de l'Enseignement supérieur, 2019) chez les élèves et les enseignants, dont celles de la collaboration et de la créativité. Car l'apprentissage du numérique s'effectue aussi par les enseignants. Dans un contexte similaire à celui des élèves, les enseignants apprennent en participant à des activités en réseau avec leur classe. II s'agit d'apprendre dans l'action, en contexte réel, ce qui facilite la pérennité des innovations et un renouvellement de la pratique plus durable (Lafortune et Deaudelin, 2001). Le développement professionnel en réseau mobilise la compétence numérique et diminue le sentiment d'isolement des enseignants (Allaire et al., 2009). L'accompagnement proximal par l'équipe de l'ÉER (coordonnateurs, collaborateurs et enseignantes ressources), la présence d'une salle de soutien pédagogique et technologique en juste à temps, les offres de formations à distance synchrones et asynchrones et les communautés de pratique (CoP) en réseau sont des propositions d'apprentissage en réseau intéressantes pour les enseignants.

Pour soutenir les enseignants de plusieurs régions du Québec dès le début du passage en enseignement à distance, une CoP a été mise en place par l'ÉER dès mars 2020. Une vingtaine d'enseignants du préscolaire et du primaire ont pu y partager leurs réflexions, bons coups et limites dans l'expérimentation de leur classe à distance et en mode hybride, ce qui a contribué au partage de pratiques novatrices, accru la pluralité de points de vue et l'expérience de contextes diversifiés. À l'automne 2020, d'autres CoP se sont organisées pour faciliter le soutien et le développement professionnel des enseignants. Les activités interclasses, le partage de ressources pédagogiques et d'expertise dans l'ÉER aident au développement de pratiques au regard du problème partagé, en l'occurrence comment mieux faire réaliser l'enseignement-apprentissage à distance. II demeure impératif de poursuivre le soutien aux enseignants dans une visée collaborative. Les modalités à explorer sont multiples, mais le besoin d'accompagnement est bien réel. 


\section{L'impact de la pandémie}

La pandémie liée à la COVID-19 a bouleversé le monde de l'éducation. Ce ne fut pas différent pour les classes déjà actives dans un mode collaboratif en réseau. Comme cité précédemment, il a été observé que les enseignants de l'ÉER ont eu plus de facilité à transformer leur enseignement dans un format à distance. II a aussi été remarqué que les activités synchrones ponctuelles, soit de courte durée, ont vu leur popularité augmenter avec l'enseignement à distance. À l'inverse, les projets de plus longue durée, qui s'étalent sur plusieurs semaines, voire plusieurs mois, ont moins suscité l'intérêt qu'auparavant. La crainte de se surcharger davantage et l'insécurité liée au contexte sont deux raisons plausibles de cette diminution de participation. Finalement, l'ÉER, avec son expertise ancrée en enseignement et apprentissage en réseau, aura su attirer l'attention de nombreux partenaires extrascolaires désirant intervenir dans la classe à distance. L'équipe de l'ÉER a su accompagner ces partenaires dans la transformation de leur offre pour la rendre dynamique et pertinente dans un mode synchrone et à distance.

\section{Conclusion}

En conclusion, l'initiative ÉER a permis d'accélérer le développement de la compétence d'enseigner à distance pour un bon nombre d'enseignants, et ce, par des activités interclasses centrées sur les apprentissages et soutenues par le numérique. L'engagement des élèves est maximisé en les plaçant au centre de leur propre réussite. Le réseau de l'ÉER procure également un accompagnement soutenu aux enseignants par d'autres enseignants, des partenaires et des membres de l'équipe de l'ÉER. La pandémie de COVID-19 a révélé les forces d'innovation, de collaboration et d'apprentissage en réseau de l'ÉER. Elle aura aussi su démocratiser l'accès à un enseignement soutenu par le numérique à tous les élèves du Québec, élèves qui auront, dans un court laps de temps, développé de nouvelles compétences numériques, de même que leurs enseignants.

\section{Liste de références}

Allaire, S., et Laferrière, T. (2013). Synthèse d'idées et de travaux à propos de la coélaboration/création de connaissances et du Knowledge Forum. Adjectif. https://constellation.uqac.ca/2690/

Allaire, S., Laferrière, T., Gaudreault-Perron, J., et Hamel, C. (2009). Le développement professionnel des enseignants en contexte de mise en réseau de petites écoles rurales géographiquement distantes : au-delà de l'alphabétisation technologique. Revue de l'apprentissage en ligne et de l'enseignement à distance, 23(3), 25-52. http://www.ijede.ca/index.php/jde/article/view/584

Allaire, S., Laferrière, T., Hamel, C., Breuleux, A., Turcotte, S., Beaudoin, J., et Inchauspé, P. (2008). L’École éloignée en réseau : Soutenir le développement professionnel des enseignants dans la mise en place de pratiques de télécollaboration en contexte d'écoles rurales. Form@re, 54, 1-7. http://affordance.uqac.ca/publications/Formare2008EERDevProfFinal.pdf

Allaire, S., et Lusignan, G. (2015). Enseigner et apprendre en réseau Guide pédagogique. https://lel.crires.ulaval.ca/sites/lel/files/allaire lusignan 2015.pdf

Bozkurt, A., Jung, I., Xiao, J., Vladimirschi, V., Schuwer, R., Egorov, G., Lambert, S. R., Al-Freih, M., Pete, J., Olcott, D., Jr., Rodes, V., Aranciaga, I., Bali, M., Alvarez, A. V., Jr., Roberts, J., Pazurek, A., Raffaghelli, J. E., Panagiotou, N., de Coëtlogon, P. ... Paskevicius, M. (2020). A global outlook to the interruption of education due to COVID-19 pandemic: Navigating in a time of uncertainty and crisis. Asian Journal of Distance Education, 15(1), 1-126. https://doi.org/10.5281/zenodo.3878572 
Bruillard, É., Ghabara, K., Huguenin, S., Jolicoeur, P.-L., Laferrière, T., Nadeau-Tremblay, S., Papi, C. et Pelletier, M.-A. (2021). L'apprentissage connecté des jeunes en contexte d'éducation formelle francophone. Revue canadienne de l'apprentissage et de la technologie, 47(4), 1-21. https://cjlt.ca/index.php/cjlt/article/view/28060/20551 .

Laferrière, T., Breuleux, A., Allaire, S., Hamel, C., Turcotte, S., Inchauspé, P., et Beaudoin, J. (2006). L'École éloignée en réseau (ÉÉR) Rapport final (Phase 2). CÉFRIO. https://eer.qc.ca/publication/1599171448197/eer-rapport-final-phase-2.pdf

Laferrière, T., Breuleux, A., et Inschauspé, P. (2004). Rapport de recherche final du projet L'École éloignée en réseau. Québec : CÉFRIO. https://eer.qc.ca/publication/1599169805412/eer-2004-rapport-de-recherche-final.pdf

Laferrière, T., Montané, M., Gros, B., Alvarez, I., Bernaus, M., Breuleux, A., Lamon, M. (2010). Partnerships for knowledge building : an emerging model / Partenariats pour la coélaboration de connaissances : un modèle en émergence. La revue canadienne de l'apprentissage et de la technologie, 36(1). https://doi.org/10.21432/T2R59Z

Lafortune, L., et Deaudelin, C. (2001). Accompagnement socioconstructiviste pour s'approprier une réforme en éducation. Presses de l'Université du Québec.

Ministère de l'Éducation et de l'Enseignement supérieur. (2019). Cadre de référence de la compétence numérique. Québec : gouvernement du Québec.

http://www.education.gouv.qc.ca/dossiers-thematiques/plan-daction-numerique/cadre-de-reference/

Ministère de l'Éducation, du Loisir et du Sport. (2018). Plan d'action numérique en éducation et en enseignement supérieur Québec : gouvernement du Québec. http://www.education.gouv.qc.ca/dossiers-thematiques/plan-daction-numerique/plan-daction-numerique/

Pelletier, M.-A., Nadeau-Tremblay, S., Bissonnette, S., Beaudoin, J. et Richard, M. (2021). La formation J'enseigne à distance : un levier pour le développement de la compétence numérique (niveaux préscolaire/primaire). Revue hybride de l'éducation, 6, 88-103. http://revues.uqac.ca/index.php/rhe/article/view/1222

Scardamalia, M., et Bereiter, C. (2010). A Brief History of Knowledge Building. La revue canadienne de l'apprentissage et de la technologie, 36(1). https://doi.org/10.21432/T2859M

Université TÉLUQ (2020). J'enseigne à distance. https://jenseigneadistance.teluq.ca/course/view.php?id=2. 